\title{
Testing light-traces-mass in Hubble Frontier Fields Cluster MACS-J0416.1-240
}

\section{Kevin Sebesta ${ }^{1}$, Liliya L. R. Williams ${ }^{1}$, Irshad Mohammed ${ }^{2,3}$ Prasenjit Saha ${ }^{2,3}$ and Jori Liesenborgs ${ }^{4}$}

\author{
${ }^{1}$ School of Physics \& Astronomy, University of Minnesota, 116 Church Street SE, Minneapolis, \\ MN 55455, USA \\ email: sebesta@physics.umn.edu, llrw@astro.umn.edu \\ ${ }^{2}$ Physik-Institut, University of Zurich, Winterthurerstrasse 190, 8057 Zurich, Switzerland \\ ${ }^{3}$ Institute for Computational Science, University of Zurich, Winterthurerstrasse 190, 8057 \\ Zurich, Switzerland \\ ${ }^{4}$ Expertisecentrum voor Digitale Media, Universiteit Hasselt, Wetenschapspark 2, B-3590, \\ Diepenbeek, Belgium
}

\begin{abstract}
We reconstruct the projected mass distribution of a massive merging HFF cluster MACSJ0416 using the genetic algorithm based free-form technique called Grale. The reconstructions are constrained by 149 lensed images identified by Jauzac et al. using HFF data. No information about cluster galaxies or light is used, which makes our reconstruction unique in this regard. Using visual inspection of the maps, as well as galaxy-mass correlation functions we conclude that overall light does follow mass. Furthermore, the fact that brighter galaxies are more strongly clustered with mass is an important confirmation of the standard biasing scenario in galaxy clusters. On the smallest scales, $\lesssim$ few arcseconds the resolution afforded by 149 images is still not sufficient to confirm or rule out galaxy-mass offsets of the kind observed in ACO 3827 . We also compare the mass maps of MACSJ0416 obtained by three different groups: Grale, and two parametric LENSTOOL reconstructions from the CATS and Sharon/Johnson teams. Overall, the three agree well; one interesting discrepancy between Grale and LENstool galaxy-mass correlation functions occurs on scales of tens of kpc and may suggest that cluster galaxies are more biased tracers of mass than parametric methods generally assume.
\end{abstract}

Keywords. Cosmology, gravitational lensing

Grale is a free-form technique of lens reconstruction that uses a genetic algorithm to calculate the mass distribution; see Liesenborgs et al. (2009) and references therein. Only the images' identifications, locations and redshifts are used as inputs for Grale.

We compare Grale results to those of two groups that use different implementation of parametric lens reconstruction method, LEnstool, CATS team and Sharon/Johnson team, which are presented in Jauzac et al. (2014) and Johnson et al. (2014), respectively, and are available for download on the HST MAST website. The CATS reconstruction is based on the HFF strong and weak lensing data, and Sharon/Johnson reconstruction uses pre-HFF strong lensing data.

The most striking, but expected difference is that LENSTOOL's galaxy-mass correlations show a pronounced spike near zero separation. This is because LENSTOOL places a lot of mass at the locations of galaxies as part of its input, whereas Grale does not.

Another notable difference is that on scales of $2^{\prime \prime}-10^{\prime \prime}$, or tens of kpc, Grale's correlation function falls less steeply than LENSTOOL's. It is possible that the lensing constraints are compatible with both the steeper and the shallower decline. However, it is also possible that the mass distribution is more extended on these scales around galaxies, as 


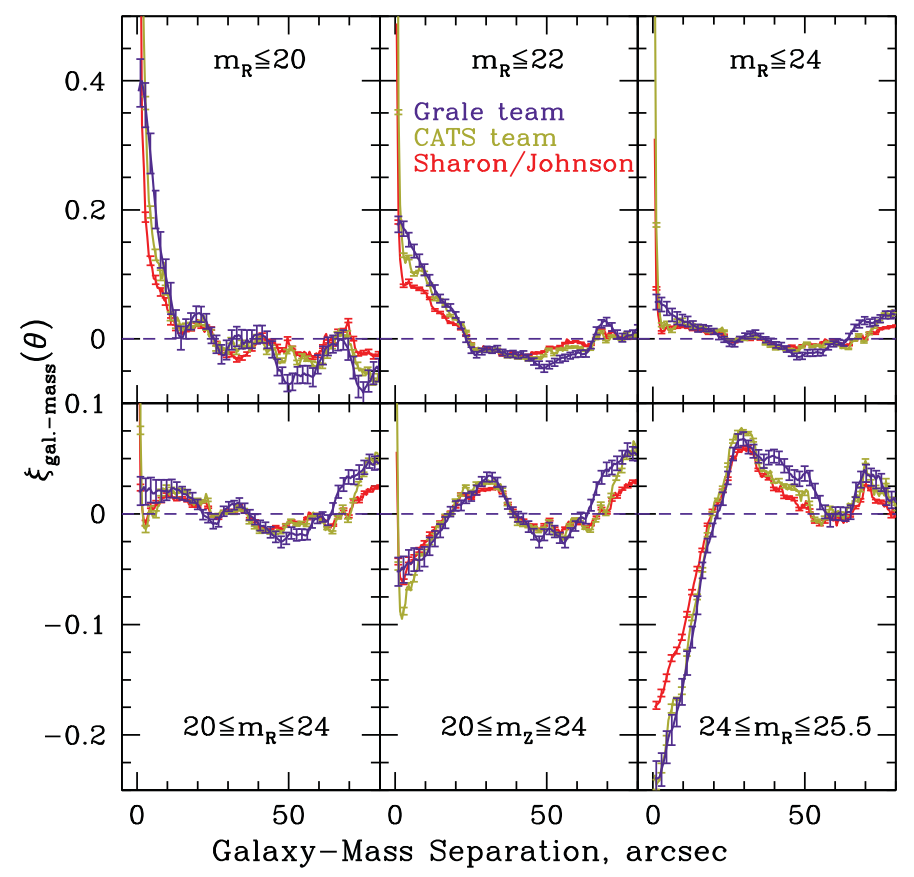

Figure 1. Galaxy-mass cross-correlation functions for five Subaru R-band and one Z-band magnitude cut. Three mass maps are used: from the Grale team (violet), CATS team (gold), and Sharon/Johnson (red). Error bars are included for every third bin to increase clarity.

Grale suggests, due to the presence of many hundreds of low surface brightness galaxies of the kind recently detected in Coma by Koda et al. (2015).

The fact that two very different methodologies - Grale and LENSTOOL-give similar results leads us to conclude that when lensed image number is around 100 or more, the images alone are sufficient to recover the mass distribution in clusters very well. Strong priors on galaxies are not needed.

Our conclusions regarding the mass vs. light distribution in MACSJ0416 are:

Overall, on scales larger than a few arcsec, light traces mass, as reconstructed by Grale, in the merging cluster MACSJ0416 quite well, as measured by the galaxy-mass correlation function. This is the only analysis of this merging cluster that does not use any information about the visible light, hence the conclusion that light follows mass is not trivial.

\section{References}

Jauzac et al. 2014, MNRAS, 443, 1549

Johnson, T. L., Sharon, K., Bayliss, M. B., Gladders, M. D., Coe, D., \& Ebeling, H. 2014, ApJ, 797,48

Koda et al. 2015, ApJL 807, L2

Liesenborgs, J., de Rijcke, S., Dejonghe, H., \& Bekaert, P. 2009, MNRAS, 397, 341 